\title{
Testing Model Structure Through a Unification of Some Modern Parametric Models of Creep: An Application to $316 \mathrm{H}$ Stainless Steel
}

\begin{abstract}
M. EVANS
It is important to be able to predict the creep life of materials used in power plants and in aeroengines. This paper develops a new parametric creep model that extends those put forward by Wilshire and Yang et al. by having them as restricted or special cases of a new generalized model. When this generalized model was applied to failure time data on $316 \mathrm{H}$ stainless steel it was found that neither of these established parametric models explained the greatest variation in the experimentally obtained times to failure. Instead, a version of this generalized model was most compatible with the experimental data. It was further found that the activation energy for this material changed at a normalized stress of 0.41 due to a change from the domination of dislocation movement within grains to movement within grain boundaries. Finally, when the generalized model was used to predict failure times beyond 5000 hours (using only the shorter test times), the new generalized model had better predictive capability at most temperatures.
\end{abstract}

https://doi.org/10.1007/s11661-019-05540-2

(C) The Author(s) 2019

\section{INTRODUCTION}

THE selection of materials for high temperature applications in power plants is typically based on the requirement that creep failure should not occur under the prevailing operating conditions during plant lives of approximately 30 years. Although complex stresses and temperatures are often encountered by materials used in power generation, design decisions are generally made on the basis of allowable tensile creep strength. This strength is commonly taken to be 67 pct of the average stress (up to $1088 \mathrm{~K}$ ). ${ }^{[1]}$ At present, protracted and expensive test programs lasting 12-15 years are necessary to determine the required long-term strengths and lives. A reduction in this $12-15$ years of 'materials development cycle' was therefore defined as the No. 1 priority in the 2007 UK Energy Materials-Strategic Research. $^{[2]}$

With the aim of reducing this development cycle, a new group of parametric creep models has been developed in recent years that are characterized through their use of a normalized stress (defined as the ratio of stress to tensile strength, $\sigma / \sigma_{\mathrm{TS}}$ ) for the determination of safe life. The rationale behind this new group of creep models is that by definition, failure will be instantaneous

M. EVANS is with the College of Engineering, Swansea University, Fabian Way, Crymlyn Burrows, Wales, SA1 8EN, UK. Contact email:m.evans@swansea.ac.uk

Manuscript submitted May 23, 2019.

Article published online November 11, 2019 when stress is equal to a material's tensile strength. Then when the material is subjected to a stress that is an infinitesimally small fraction of its tensile strength, the material should remain intact for a very long period of time. That is, $\mathrm{t}_{\mathrm{f}}$ must vary from 0 towards $\infty$ as $\sigma / \sigma_{\mathrm{TS}}$ varies from 1 towards 0 . Unfortunately, the rate at which this happens is not fully understood, and so this group of models assumes that the relationship between $\sigma / \sigma_{\mathrm{TS}}$ and $t_{\mathrm{f}}$ (at a fixed temperature) is given by an inverted S-shaped curve. ${ }^{[3,4]}$ The models within the group are then differentiated by the mathematical function used to describe this inverted S-shaped curve, and so consequently these models can end up producing very different safe life predictions.

This paper aims to tackle this problem by specifying a generalized model that nests the creep model first put forward by Wilshire and Battenbough ${ }^{[3]}$ and the model proposed by Yang et al. ${ }^{[4]}$ i.e., these two models are special cases within this more general model. Within such a framework, it is then possible to use some basic statistical tests to allow experimental creep data to determine the correct shape of the inverted S-shaped relationship between $\sigma / \sigma_{\mathrm{TS}}$ and $t_{\mathrm{f}}$. Once identified, this shape can be used to obtain safe life predictions that are compatible with experimental data, rather simply using some ad hoc functional form for the S-shaped relationship. To achieve this aim, the paper is structured as follows. The next section gives a brief review of old and new parametric creep models and this is followed by a section deriving the generalized creep model together with a statistical test for the form of the inverted S-shaped curve. The next section then estimates the 
unknown parameters of these models together with a short discussion on likely failure mechanisms that could account for the values of these estimated parameters. There then follows a subsection comparing the predictive accuracy of the Wilshire, Yang et al. and generalized creep models when they are applied to experimentally derived failure times for $316 \mathrm{H}$ stainless steel. The paper ends with a conclusion section that outlines some areas for future work.

\section{PARAMETRIC CREEP MODELS}

\section{A. A Brief History}

The basis for most of the existing parametric models used for creep life prediction is the modification of an equation put forward by Eyring ${ }^{[5]}$ for explaining a rate constant in the presence of both thermal and non-thermal stresses. When studying creep, the chosen rate constant is usually taken to be the minimum creep rate, $\dot{\varepsilon}_{\mathrm{m}}$

$$
\dot{\varepsilon}_{\mathrm{m}}=A T^{\alpha} \exp \left\{-\frac{Q_{\mathrm{c}}}{R T}+B f(\sigma)+D \frac{f(\sigma)}{T}\right\},
$$

where $T$ is temperature, $\sigma$ stress, and $Q_{\mathrm{c}}$ is the activation energy for self-diffusion. $\alpha, A, B$, and $D$ are model parameters whose values are usually unknown. The form of the stress function, $f(\sigma)$, however, is application specific and it explains the variety of parametric creep models present in the engineering literature. An equation for the time to failure, $t_{\mathrm{f}}$, can then be obtained from this by making use of the empirical Monkman-Grant ${ }^{[6]}$ relation that contains further constants $M$ and $\rho$ (where $\rho$ is close to unity in value for many metal alloys):

$$
\dot{\varepsilon}_{\mathrm{m}}=\left(M / t_{\mathrm{f}}\right)^{\rho}
$$

so that Eq. [1a] can be rewritten as

$$
\ln \left(t_{\mathrm{f}}\right)=a_{0}+a_{1} f(\sigma)+a_{2} \ln (T)+a_{3} \frac{1}{T}+a_{4} \frac{f(\sigma)}{T},
$$

where $\mathrm{a}_{0}=\{-\ln (A) / \rho+\ln (M)\}, a_{1}=-B / \rho, a_{2}=-\alpha /$ $\rho, a_{3}=Q_{\mathrm{c}} /(\rho R)$, and $a_{4}=-D / \rho$.

The so-called first Soviet model ${ }^{[7]}$ is very similar to Eq. [1c] in that $\mathrm{f}(\sigma)=\ln (\sigma)$ for the expression in front of parameter $a_{1}$, but $f(\sigma)=\sigma$ in the expression in front of $a_{4}$. Although Larson-Miller ${ }^{[8]}$ never specified a specific stress function in their paper, the model is often represented has having $a_{1}=a_{2}=0$ with $f(\sigma)=\ln (\sigma)$ and the Orr-Sherby-Dorn ${ }^{[9]}$ model has $a_{2}=a_{4}=0$ with $f(\sigma)=\ln (\sigma)$. The Minimum Commitment Method $^{[10]}$ has $a_{4}=0$ and uses $T$ instead of $\ln (T)$ with $f(\sigma)=\ln (\sigma)+b_{1} \sigma+b_{2} \sigma^{2}$, where $b_{1}$ and $b_{2}$ are additional model parameters. Finally, Manson and Haferd $^{[11]}$ set $a_{2}=a_{3}=0$ and replace $a_{4} f(\sigma) / T$ with $a_{4} f(\sigma)\left[T-a_{5}\right]$, while Manson and Brown ${ }^{[12]}$ further generalize using $a_{4} f(\sigma)\left[T-a_{5}\right]^{a 6}$, where $a_{5}$ and $a_{6}$ are additional model parameters (with $f(\sigma)$ being some polynomial function of stress). Table I summarizes these competing models expressing rupture life as an algebraic function of stress and temperature. In recent years, a newer group of parametric models has found their way into the literature. They are different to those described above as they emphasis the role of the normalized stress in the determination of failure times, $\sigma / \sigma_{\mathrm{TS}}$, where $\sigma_{\mathrm{TS}}$ is the tensile strength of the material under investigation. Two examples of this group of models are the Wilshire ${ }^{[3]}$ equations $\left(a_{2}=a_{4}=0\right.$ with $f(\sigma)=\ln \left[-\ln \left(\sigma / \sigma_{\mathrm{TS}}\right)\right]$ in Eq. [1c]) and the model by Yang et al. ${ }^{[4]}\left(a_{2}=a_{4}=0\right.$ with $f(\sigma)=\ln \left(\sigma /\left(\sigma_{\mathrm{TS}}-\sigma\right)\right)$ in Eq. [1c]).

\section{B. Normalized Stress Creep Models}

\section{Isothermal}

At a given temperature, the approach towards modeling stress taken by the more modern parametric techniques is through the use of the normalized stress $\sigma / \sigma_{\mathrm{TS}}$. The rationale behind this new group of creep models is that by definition, failure will be instantaneous when stress is equal to a material's tensile strength. Then when the material is subjected to a stress that is an infinitesimally small fraction of its tensile strength, the material should remain intact for a very long period of time. Unfortunately, the rate at which this happens is not really known, and the modern parametric techniques assume that the relationship between $\sigma / \sigma_{\mathrm{TS}}$ and $t_{\mathrm{f}}$ (at a fixed temperature) is given by an inverted S-shaped curve. Thus, in the model by Yang et al., ${ }^{[4]}$ this inverted $\mathrm{S}$-shaped function takes the form

$$
\frac{\sigma}{\sigma_{\mathrm{TS}}}=\left[1+\left(\frac{t_{\mathrm{f}}}{\alpha_{\mathrm{L}}}\right)^{\beta_{\mathrm{L}}}\right]^{-1},
$$

where $\alpha_{\mathrm{L}}$ and $\beta_{\mathrm{L}}$ are model parameters (constants). $\alpha_{\mathrm{L}}$ is termed a scale parameter because its value equals the failure time associated with a normalized stress of 0.5 and this point on the S-shaped curve also corresponds to the inflection point. $\beta_{\mathrm{L}}$ is termed the shape parameter as its value determines how flat the $\mathrm{S}$ shaped curve is around the inflection point $\left(\beta_{\mathrm{L}}\right.$ values close to 2 result in a very flat $S$ shape that is almost linear in nature). These characteristics can be seen in Figure 1(a). Many readers with a statistical background may recognize this equation as the same as that describing the Logistic cumulative distribution function. This can be rewritten in terms of the time to failure itself

$$
\ln \left[t_{\mathrm{f}}\right]=\ln \left[\alpha_{\mathrm{L}}\right]+\frac{1}{\beta_{\mathrm{L}}} \ln \left[\frac{1}{r}-1\right],
$$

where $r=\sigma / \sigma_{\mathrm{TS}}$. Given that $\ln \left[\frac{1}{r}-1\right]=-\ln \left[\frac{r}{1-r}\right]$, Eq. [1b] can be written in the way initially specified by Yang et al.,

$$
\ln \left[t_{\mathrm{f}}\right]=\ln \left[\alpha_{\mathrm{L}}\right]-\frac{1}{\beta_{\mathrm{L}}} \ln \left[\frac{r}{1-r}\right]
$$

In the model by Wilshire, ${ }^{[3]}$ this inverted S-shaped function takes the form 
Table I. Summary of Some Parametric Creep Models for Predicting Time to Failure

Model Name

Orr-Sherby-Dorn ${ }^{[9]}$ (OSD)

Larson-Miller ${ }^{[8]}$ (LM)

Minimum Commitment ${ }^{[10]}(\mathrm{MC})$

Manson and Haferd ${ }^{[11]}(\mathrm{MH})^{*}$

Manson and Brown ${ }^{[12]}(\mathrm{MB})^{*}$

Soviet Model $1^{[7]}$ (SM1)

Wilshire $^{[3]}(\mathrm{W})$

Yang et al. ${ }^{[4]}(\mathrm{Y})$
Single Comprehensive Equation

$$
\begin{aligned}
& \ln \left(t_{\mathrm{f}}\right)=a_{0}+a_{1} \ln (\sigma)+a_{3} \frac{1}{T} \\
& \ln \left(t_{\mathrm{f}}\right)=a_{0}+a_{3} \frac{1}{T}+a_{4} \frac{\ln (\sigma)}{T} \\
& \ln \left(t_{\mathrm{f}}\right)=a_{0}+a_{1} \ln (\sigma)+a_{2} T+a_{3} \frac{1}{T}+b_{1} \sigma+b_{1} \sigma^{2} \\
& \ln \left(t_{\mathrm{f}}\right)=a_{0}+a_{1} f(\sigma)+a_{4} \frac{f(\sigma)}{T}\left[T-a_{5}\right] \\
& \ln \left(t_{\mathrm{f}}\right)=a_{0}+a_{1} f(\sigma)+a_{4} \frac{f(\sigma)}{T}\left[T-a_{5}\right]_{6} \\
& \ln \left(t_{\mathrm{f}}\right)=a_{0}+a_{2} \ln (\sigma)+a_{3} \frac{1}{T}+a_{4} \frac{\sigma}{T} \\
& \ln \left(t_{\mathrm{f}}\right)=a_{0}+a_{1} \ln \left[-\ln \left(\sigma / \sigma_{\mathrm{TS}}\right)\right],+a_{3} \frac{1}{T} \\
& \ln \left(t_{\mathrm{f}}\right)=a_{0}+a_{1} \ln \left[\sigma /\left(\sigma_{\mathrm{TS}}-\sigma\right)\right]+a_{3} \frac{1}{T}
\end{aligned}
$$

*In these models, $f(\sigma)$ is a polynomial function of stress. In stands for natural log, $t_{\mathrm{f}}$ is the time to failure, $T$ the test temperature, $\sigma$ the test stress, and $\sigma_{\mathrm{TS}}$ the tensile strength. $a_{0}$ to $a_{6}$ are model parameters that require estimation and have different meanings and values in each of the models.
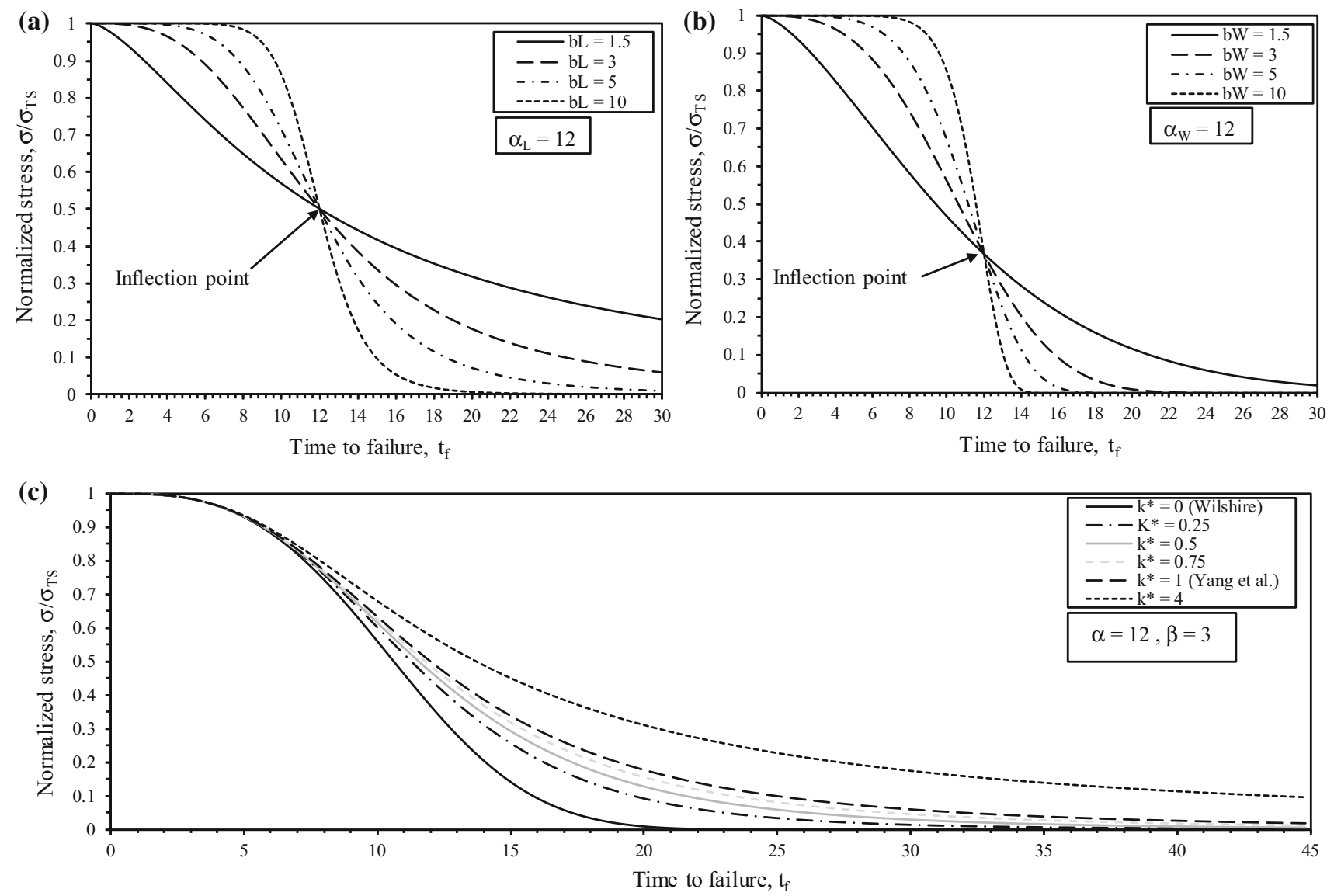

Fig. 1-Showing the form of (a) Yang et al's. model for different values $\beta_{\mathrm{L}}$ when $\alpha_{\mathrm{L}}=12$ and, (b) Wilshire's model for different values of $\beta_{\mathrm{W}}$ when $\alpha_{\mathrm{L}}=12,(c)$ generalized model for different values of $k^{*}$ when $\alpha=12$ and $\beta=3$.

$$
\frac{\sigma}{\sigma_{\mathrm{TS}}}=\exp \left[-\left(\frac{t_{\mathrm{f}}}{\alpha_{\mathrm{W}}}\right)^{\beta_{\mathrm{W}}}\right],
$$

where $\alpha_{\mathrm{W}}$ and $\beta_{\mathrm{W}}$ are model parameters (again some may recognize this equation as that describing the Weibull cumulative distribution function). The value for $\alpha_{\mathrm{W}}$ equals the failure time associated with a normalized stress of 0.368 and this point on the S-shaped curve also corresponds to the inflection point. $\beta_{\mathrm{L}}$ is termed the shape parameter as its value determines how flat the $\mathrm{S}$ shaped curve is around the inflection point $\left(\beta_{\mathrm{L}}\right.$ values close to 1.25 result in a very flat $\mathrm{S}$ shape that is almost linear in nature and a value close to 5 results in a very step but approximately symmetric $\mathrm{S}$ shape around the inflection point). These 
characteristics can be seen in Figure 1(b). Again this can be rewritten in terms of the time to failure

$$
\ln \left[t_{\mathrm{f}}\right]=\ln \left[\alpha_{\mathrm{W}}\right]+\frac{1}{\beta_{\mathrm{W}}} \ln [-\ln (r)] .
$$

Given that the exact shape of the function linking $\sigma /$ $\sigma_{\mathrm{TS}}$ to $t_{\mathrm{f}}$ is unknown, it is advantageous to formulate a more flexible inverted $\mathrm{S}$-shaped curve that contains the above two models as special cases. Put differently, each model above assumes the inflection point occurs at a specific normalized stress and the actual creep data may not display this characteristic. By creating a generalized model, it then becomes possible to create a statistical test to determine the nature of the relationship between $\sigma$ / $\sigma_{\mathrm{TS}}$ and $t_{\mathrm{f}}$ that is most supported by the experimental creep data. Generalization can be achieved by introducing an extra parameter $\mathrm{k}$ into $\mathrm{Eq}$. [2a] such that when $k=1$ the model by Yang et al. is obtained and when $k$ becomes very large the model by Wilshire emerges. For $k$ values in between, completely new models emerge with a range of different $\mathrm{S}$-shaped curves. This paper proposes the following generalized model that nests the above two models through the introduction of an additional parameter $\mathrm{k}$ that has inflection points occurring at differing normalized stresses depending on the value for $k$

$$
\frac{\sigma}{\sigma_{\mathrm{TS}}}=\left[1+\frac{1}{k}\left(\frac{t_{\mathrm{f}}}{\alpha}\right)^{\beta}\right]^{-k}
$$

or in terms of the time to failure

$$
\ln \left[t_{\mathrm{f}}\right]=\ln [\alpha]+\frac{1}{\beta} r^{*}
$$

where

$$
r^{*}=\ln \left[k\left(\frac{\sigma}{\sigma_{\mathrm{TS}}}\right)^{-1 / k}-k\right]
$$

Now, when $k=1, r^{*}=\ln \left[\frac{1}{r}-1\right]$, and so Eqs. [4a] and [4b], simplify into Eqs. [2a] and [2b] with $\alpha=\alpha_{\mathrm{L}}$ and $\beta=\beta_{\mathrm{L}}$. Thus $k=1$ results in the model proposed by Yang et al. Further, as $k \rightarrow \infty, r^{*} \rightarrow \ln [-\ln (r)]$ and so then Eqs. [4a] and [4b], simplify into Eqs. [3a] and [3b] with $\alpha=\alpha_{\mathrm{W}}$ and $\beta=\beta_{\mathrm{W}}$. Thus, $\mathrm{k}$ tending to $\infty$ results in the model by Wilshire. Other inverted S-shaped curves exist for $\mathrm{k}$ values between these two limits. The different inverted S-shaped curves associated with the different values for $k$ are shown in Figure 1(c), where $\mathrm{k}$ is rescaled to fall within the limits 0 to 1 through the transformation $k^{*}=k^{-0.5}$ (so that when $k=1, \mathrm{k}^{*}=1$ and as $k \rightarrow$ $\left.\infty, k^{*} \rightarrow 0\right)$. In this rescaling, Yang et al.'s model corresponds to $k^{*}=1$ and Wilshire's model emerges when $k^{*}=0$. Figure $1(\mathrm{c})$ shows the different shapes produced by different values for $k^{*}$ in Eq. [4c] when $\beta=$ 3 and $\alpha=12$.

\section{Temperature compensated}

If the effect of temperature on creep life is determined by the Arrhenius equation, the role of the reciprocal of temperature is simply to alter the value for the intercept term in Eq. [4b]

$$
\ln \left[t_{\mathrm{f}}\right]=\left\{\ln [\alpha]+Q_{c} \frac{1}{R T}\right\}+\frac{1}{\beta} r^{*},
$$

where $R$ is the universal gas constant. Rearranging Eq. [5a] for $r=\sigma / \sigma_{\mathrm{TS}}$ gives

$$
r=\frac{\sigma}{\sigma_{\mathrm{TS}}}=\left[1+\frac{1}{k \alpha^{\beta}}\left(t_{\mathrm{f}} \exp \left\{-Q_{c} \frac{1}{R T}\right\}\right)^{\beta}\right]^{-k}
$$

Again, Yang et al.'s model corresponds to $k^{*}=1$ and Wilshire's model emerges when $k^{*}=0$.

The Wilshire and Yang et al. models have been applied to a wide range of materials and in all cases there is a need for partitioning, in the sense that the model parameters only remain constant over a limited range of normalized stress values

$$
\ln \left[t_{\mathrm{f}}\right]=\left\{\ln \left[\alpha_{j}\right]+Q_{\mathrm{cj}} \frac{1}{R T}\right\}+\frac{1}{\beta_{j}} r^{*}
$$

$j=1$ when $\sigma / \sigma_{\mathrm{TS}} \leq \sigma_{1}^{\mathrm{c}} ; j=2$ when $\sigma_{1}^{\mathrm{c}}<\sigma / \sigma_{\mathrm{TS}} \leq \sigma_{2}^{\mathrm{c}} ; \ldots ; j=p$ when $\sigma / \sigma_{\mathrm{TS}}>\sigma_{p-1}^{\mathrm{c}}$

$$
\sigma_{1}^{\mathrm{c}}<\sigma_{2}^{\mathrm{c}}<\cdots<\sigma_{p-1}^{\mathrm{c}}
$$

$\sigma_{\mathrm{j}}^{\mathrm{c}}$ are critical values for the normalized stresses and so fall between 0 and 1 . In partitioning, there are $p$ creep regimes that occur in distinct ranges for the normalized stress and the $\mathrm{p}$ versions of Eq. [5c] then apply to each regime. Typically, $p$ varies between 1 and 4 depending on the material being studied and the test conditions present in the creep data base on that material. Some of the first studies to appear in the literature include applications to Copper ${ }^{[3]}$ and $1 \mathrm{Cr}-1 \mathrm{Mo}-0.25$ stee $^{[13]}$ where $\mathrm{p}$ was found equal to 2 and where the activation energy was the same either side of $\sigma_{1}^{\mathrm{c}}$ ( so $Q_{\mathrm{cj}}=Q_{c}$ ). Later studies by Whittaker and Wilshire using $2.25 \mathrm{Cr}-1 \mathrm{Mo}$ steel $^{[14]}$ found $\mathrm{p}$ equal to 3 and again the activation energy was the same either side of $\sigma_{1}^{\mathrm{c}}$ and $\sigma_{2}^{\mathrm{c}}$ and Evans ${ }^{[15]}$ found $p=2$ but with a varying activation energy either side of $\sigma_{1}^{\mathrm{c}}$ when studying a $12 \mathrm{Cr}$ steel. Finally, Whittaker et al. ${ }^{[16]}$ found $p=2$ with varying activation energies around $\sigma_{1}^{\mathrm{c}}$ when studying a particular grade of Waspaloy.

\section{Estimation and a statistical test for the stress} function (i.e., for the value for $k$ )

As Eq. [5b] contains a number of unknown parameters, including the activation energy, data are required on the time taken for specimens tested under different stresses and temperatures to fail. A number of large 
creep data sets of this nature exist and what is clear from them is that failure times exhibit variation over and above that explainable from test conditions alone. To account for this stochastic nature of creep failure times, a random error term, e, whose standard deviation is given by $\tau$, can be added to Eqs. [5a] and [5c]

$$
\ln \left[t_{\mathrm{f}}\right]_{i}=\ln [\alpha]+Q_{c}\left[\frac{1}{R T_{i}}\right]+\frac{1}{\beta} r_{i}^{*}+e_{i}
$$

or with partitioning

$$
\ln \left[t_{\mathrm{f}}\right]_{i}=\ln \left[\alpha_{j}\right]+Q_{\mathrm{cj}}\left[\frac{1}{R T_{i}}\right]+\frac{1}{\beta_{j}} r_{i}^{*}+e_{i} \quad j=1, p
$$

where the subscript $i$ designates the failure time of the ith specimen tested at temperature $T_{i}$ and transformed stress $r_{i}^{*} \cdot e_{i}$ is then the component of $\ln \left[t_{\mathrm{f}}\right]_{i}$ that cannot be explained by the stress and temperature experienced by the ith specimen. Given this, it makes sense to select values for the unknown parameters in Eqs. [6a] and [6b] so as to minimize this random or unexplained variation. The well-known least squares procedure does exactly this - it makes use of simple equations that attach values to $Q_{\mathrm{c}}, \alpha$, and $\beta$ so as to minimize the sum of squared errors, $\sum \mathrm{e}_{\mathrm{i}}^{2}$, where this summation is over all $i=1$ to $n$ observations on $t_{\mathrm{f}}$. To minimize the sum of squared errors, a value for $k$ must first be chosen so that $r_{i}^{*}$ can be calculated using Eq. [4c]. For the unpartitioned version, all these parameters are estimated by a regression of $\ln \left[t_{\mathrm{f}}\right]$ on $r^{*}$ and $1 / R T$. This regression will have an associated coefficient of determination $\left(R^{2}\right)$ - that shows what percentage of the variation in $\ln \left[t_{\mathrm{f}}\right]$ can be explained by variations in all the test variables on the right-hand side of Eq. [6a]. The values for $\ln (\alpha), \beta$, and $Q_{\mathrm{c}}$ obtained in this way are designated through the use of a hat symbol, i.e., $\hat{\alpha}, \hat{\beta}$ and $\hat{Q}_{\mathrm{c}}$ are the least squares estimates of $\ln (\alpha), \beta$, and $Q_{\mathrm{c}}$.

For the partitioned version when $p=2$, estimation first requires the construction of a dummy variable $\mathrm{D}$ by initially estimating (by eye) a value for $\sigma_{1}^{\mathrm{c}}$

$$
\begin{aligned}
\ln \left[t_{\mathrm{f}}\right]_{i}= & \ln \left[\alpha_{1}\right]+Q_{c 1}\left[\frac{1}{R T_{i}}\right]+\frac{1}{\beta_{1}} r_{i}^{*}+\delta_{0} D_{i}+\delta_{1}\left[r_{i}^{*} D_{i}\right] \\
& +\delta_{2}\left[\frac{D_{i}}{R T_{i}}\right]+e_{i},
\end{aligned}
$$

where the dummy variable $D=0$ when $\sigma / \sigma_{\mathrm{TS}}>\sigma_{1}^{\mathrm{c}}$ but $D=1$ otherwise. Thus when $j=2$ because $\sigma / \sigma_{\mathrm{TS}}>\sigma_{1}^{\mathrm{c}}$, Eq. [7a] collapses to

$$
\ln \left[t_{\mathrm{f}}\right]_{i}=\ln \left[\alpha_{1}\right]+Q_{c 1} \frac{1}{R T_{i}}+\frac{1}{\beta_{1}} r_{i}^{*}+e_{i} .
$$

Then when $j=1$, because $\sigma / \sigma_{\mathrm{TS}} \leq \sigma_{1}^{\mathrm{c}}, D=1$ and Eq. [7a] can be re-arranged as

$$
\begin{aligned}
\ln \left[t_{\mathrm{f}}\right]_{i}= & \left\{\ln \left[\alpha_{1}\right]+\delta_{0}\right\}+\left\{Q_{c 1}+\delta_{2}\right\} \frac{1}{R T_{i}}+\left\{\frac{1}{\beta_{1}}+\delta_{1}\right\} r_{i}^{*} \\
& +e_{i}
\end{aligned}
$$

and so $Q_{\mathrm{c} 2}=Q_{\mathrm{c} 1}+\delta_{2}, 1 / \beta_{2}=1 / \beta_{1}+\delta_{1}$, and $\ln \left[\alpha_{2}\right]=$ $\ln \left[\alpha_{1}\right]+\delta_{0}$. All these parameters are estimated by a regression of $\ln \left[t_{\mathrm{f}}\right]$ on $r^{*}, 1 / R T, D, r^{*} D$, and $\left[\frac{D}{R T}\right]$ once a value of $k$ has been chosen. This regression will have an associated coefficient of determination $\left(R^{2}\right)$ - that shows what percentage of the variation in $\ln \left[t_{\mathrm{f}}\right]$ that can be explained by variations in all the variables on the right-hand side of Eq. [7c]. The values for $\ln \left(\alpha_{1}\right), \ln \left(\alpha_{2}\right)$, $\beta_{1}, \beta_{2}, Q_{\mathrm{c} 1}$, and $Q_{\mathrm{c} 2}$ obtained in this way are designated through the use of a hat symbol, i.e., $\hat{\alpha}_{1}, \hat{\alpha}_{2}, \hat{\beta}_{1}, \hat{\beta}_{2}, \hat{Q}_{\mathrm{cl} 1}$ and $\hat{Q}_{\mathrm{c} 1}$ are least squares estimates of $\ln \left(\alpha_{1}\right), \ln \left(\alpha_{2}\right), \beta_{1}, \beta_{2}, Q_{\mathrm{c} 1}$, and $Q_{\mathrm{c} 2}$. This regression is carried out for all values of $\sigma_{1}^{\mathrm{c}}$ within the experimental range of normalized stresses and the value $\sigma_{1}^{\mathrm{c}}$ is taken to be that value which gives the largest $R^{2}$ value.

Further, minimizing the sum of the squared errors is equivalent to maximizing the joint probability or likelihood of observing all $n$ failure times, provided the random errors (and thus the logged times to failure) follow a normal distribution. Given this assumption, the $\log$ of this joint probability, termed the log likelihood, can be calculated as

$$
\ln \left(L_{k}\right)=-\frac{n}{2} \ln (2 \pi)-\frac{n}{2} \ln \left(\hat{\tau}^{2}\right)-\frac{1}{2 \hat{\tau}^{2}} \sum_{i=1}^{n} \hat{e}_{i}^{2},
$$

where without partitioning

$$
\hat{e}_{i}^{2}=\left\{\ln \left[t_{\mathrm{f}}\right]_{i}-\left[\ln [\hat{\alpha}]+\hat{Q}_{c} \frac{1}{R T_{i}}+\frac{1}{\hat{\beta}} r_{i}^{*}\right]\right\}^{2}
$$

and with partitioning (when $p=2$ )

$\hat{e}_{i}^{2}=\left\{\ln \left[t_{\mathrm{f}}\right]_{i}-\ln \left[\hat{\alpha}_{1}\right]-\hat{Q}_{c 1} \frac{1}{R T_{i}}-\frac{1}{\hat{\beta}_{1}} r_{i}^{*}-\hat{\delta}_{0} D_{i}-\hat{\delta}_{1}\left[r_{i}^{*} D_{i}\right]-\hat{\delta}_{2}\left[\frac{D_{i}}{R T_{i}}\right]\right\}^{2}$.

In both cases,

$$
\hat{\tau}^{2}=\frac{\sum_{i=1}^{n} \hat{e}_{i}^{2}}{n-\text { number of estimated parameters }},
$$

where $n$ equals the total number of tests in the data set. Such a value for this log likelihood can be calculated for all values for $\mathrm{k}, \operatorname{Ln}\left(L_{\mathrm{k}}\right)$, and there will be some value for $k$ that results in the largest log likelihood, $\operatorname{Ln}\left(L_{\mathrm{k}}^{+}\right)$. The test statistic (or relative $\log$ likelihood)

$$
\chi^{2}=2\left[\operatorname{Ln}\left(L_{k}^{+}\right)-\operatorname{Ln}\left(L_{k}\right)\right]
$$

has a $\chi^{2}$ distribution with one degree of freedom under the null hypothesis that $\left(k^{+}-k\right)$ equals zero. $k^{+}$is thus 
the value for $k$ most supported by the data, and values for $\mathrm{k}$ that lead to $\chi^{2}>3.814$ are not supported by the failure time data at the 5 pct significance level.

\section{THE DATA}

The NIMS Matnavi website, ${ }^{[17]}$ in which Creep Data Sheet 14B resides, provides extensive rupture data for two batches (labeled $\mathrm{AaA}$ and $\mathrm{AaB}$ ) of $316 \mathrm{H}$ stainless steel plate $\left(18 \mathrm{Cr}-12 \mathrm{Ni}-\mathrm{Mo}\right.$ with up to $\left.0.08{ }^{\circ} \mathrm{C}\right)$. These two plate specimens were hot rolled and held at $1323 \mathrm{~K}$ for either 40 or 80 minutes before water quenching and each batch had a different chemical composition which can be found in Creep Data Sheet 14B. ${ }^{18]}$ This paper uses both batches and so is made up of 60 specimens that were tested at nine different temperatures ranging from $873 \mathrm{~K}$ to $1123 \mathrm{~K}$, and stresses ranging from 265 to $20 \mathrm{MPa}$. The resulting shortest failure time was 175, 320 seconds and the largest was 469, 720, 440 seconds (or approximately 15 years). Figure 2 shows all the normalized stresses and temperatures applied to the test specimens together with the time at which each specimen failed and Table II shows the temperature dependency of this material's tensile strength as well as its batch to batch variability.

\section{RESULTS}

\section{A. The Wilshire Model $\left(k^{*}=0\right)$}

Whittaker et al. ${ }^{[19]}$ carried out a detailed study of this material using the Wilshire model and found there to be two distinct creep regimes $(p=2)$. This finding was obtained using steel tubes, plate, and piping material rather than just the tube data used in this paper. Sticking with this finding, and using the estimation techniques described above when $k^{*}=0$ (so leading to the Wilshire model), resulted in the largest $R^{2}$ value being associated with $\sigma_{1}^{\mathrm{c}}=0.41$ and this is similar in value to that found by Whittaker et al. The resulting least squares estimates of the models parameters were then found to be

$$
\text { If } \sigma / \sigma_{\mathrm{TS}}>0.41
$$

$\ln \left[t_{\mathrm{f}}\right]=\underset{\{-9.23\}}{-22.532}+7.543 \ln \left[-\ln \left(\sigma / \sigma_{\mathrm{TS}}\right)\right]+306, \underset{\{14.99\}}{131[1 / R T]}$.

$$
\begin{aligned}
& \text { If } \sigma / \sigma_{\mathrm{TS}} \leq 0.41 \\
& \ln \left[t_{\mathrm{f}}\right]=\underset{\{-2.21\}}{-8.025}+\underset{\{10.03\}}{7.377} \ln \left[-\ln \left(\sigma / \sigma_{\mathrm{TS}}\right)\right]+\underset{\{6.33\}}{181,573[1 / T]}
\end{aligned}
$$

The parameter estimates shown in Eq. [10] imply that when $\sigma / \sigma_{\mathrm{TS}}>0.41, \alpha_{\mathrm{w}}=\exp (-22.532)=1.6386 \mathrm{E}-10$, $\beta_{\mathrm{w}}=1 / 7.543=0.1356$ with an activation energy of 306 $\mathrm{kJmol}^{-1}$. But at a normalized stress below 0.41 the activation energy drops to $182 \mathrm{kJmol}^{-1}$ with $\alpha_{\mathrm{w}}=$ $\exp (-8.025)=0.0003$ and $\beta_{\mathrm{w}}=1 / 7.377=0.1355$. The estimated parameters shown in Eq. [10] are based on all the experimental failure times and the numbers in squiggly brackets below these estimated values are the student $t$ values associated for testing the null hypothesis that the population parameter values are really zero. Consequently, all the parameters are statistically significant at the 5 pct significance level. The student $t$ values for $\delta_{0}$ and $\delta_{2}$ also revealed that both these parameters are also significantly different from zero at the 5 pct

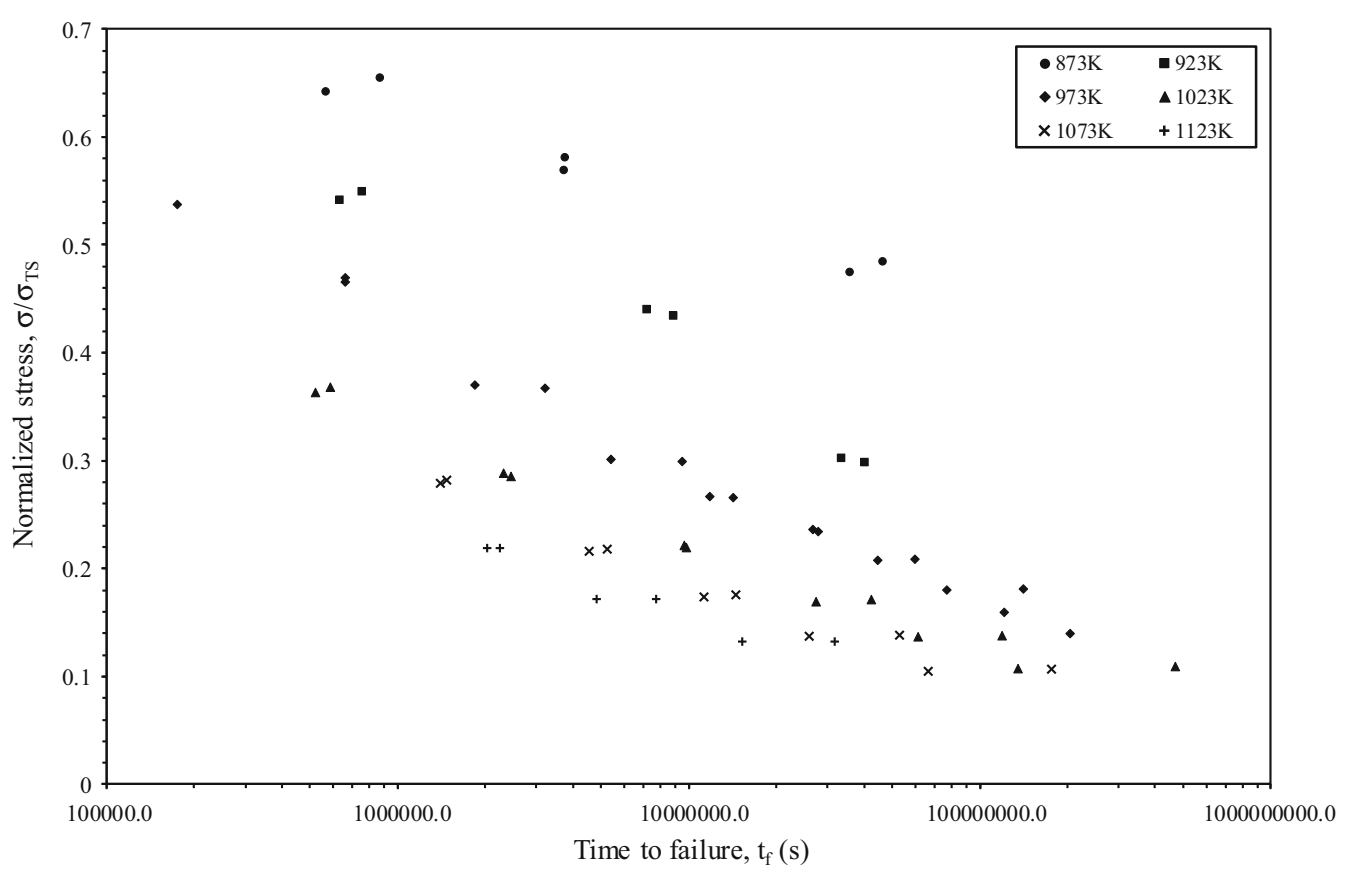

Fig. 2-Plot of time to failure against the normalized stress at various temperatures for all batches of $316 \mathrm{H}$ austenitic stainless steel taken from the NIMS creep data sheet 14B. 
significance level, but that the student $\mathrm{t}$ value for $\delta_{1}$ revealed that this parameter was not significantly different from zero at the same significance level. Consequently, at a normalized stress of 0.41 there is a real change in the value for $\alpha_{\mathrm{w}}$ and the activation energy, but no change in the slope parameter $\beta \mathrm{w}$. This structure is shown visually in Figure 3(a), where the fitted line has essentially the same slope over the full range of stresses. The overall $R^{2}$ value was 96.62 pct.

Table II. Variation of Tensile/0.2 Pet Proof Stress (MPa) with Temperature (K) and Batch

\begin{tabular}{lcc}
\hline Temperature & Batch AaA & Batch AaB \\
\hline 873 & $413 / 120$ & $405 / 114$ \\
923 & $362 / 123$ & $357 / 128$ \\
973 & $294 / 109$ & $292 / 116$ \\
1023 & $242 / 110$ & $239 / 108$ \\
1073 & $190 / 92$ & $188 / 101$ \\
1123 & $151 / 85$ & $151 / 92$ \\
\hline
\end{tabular}
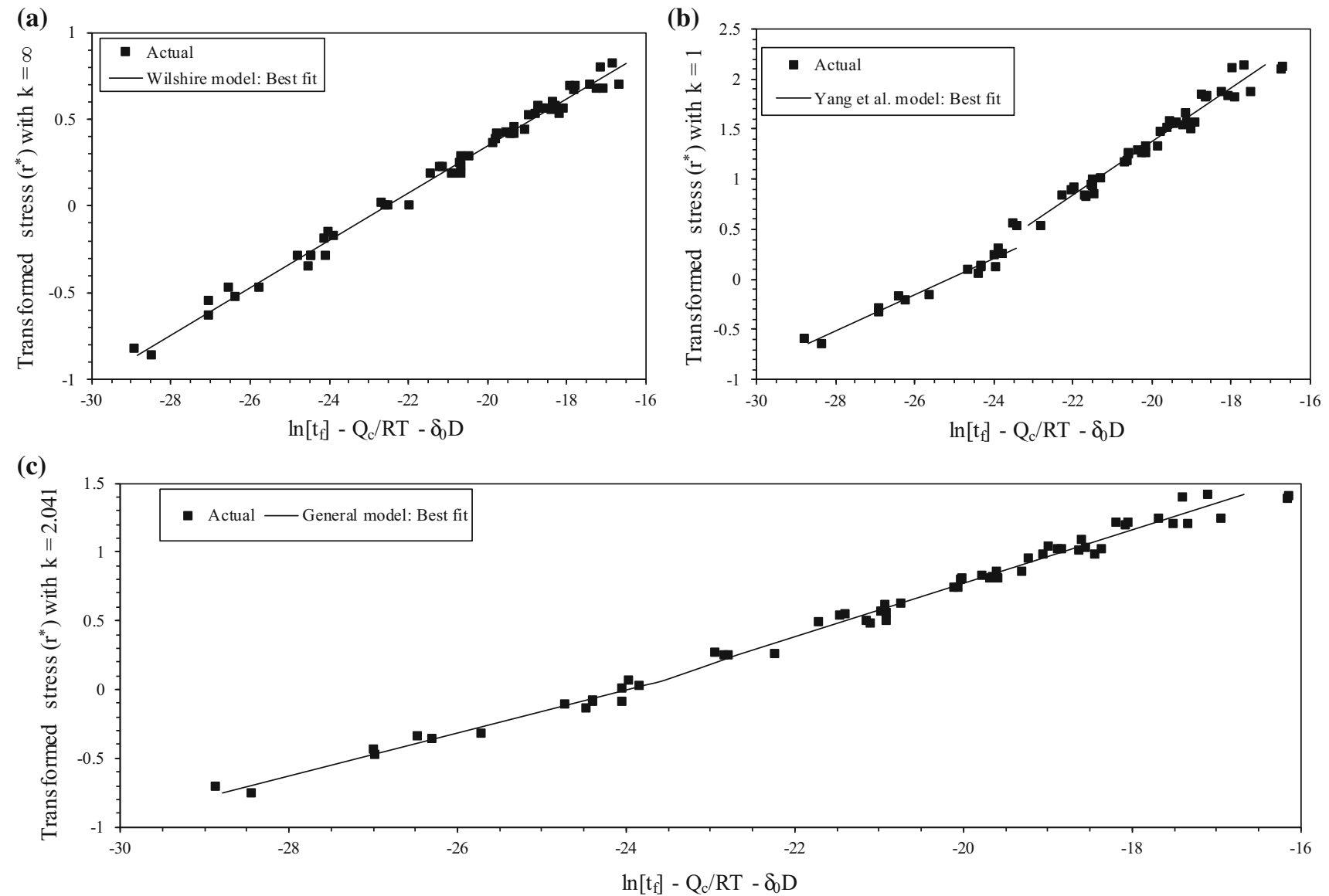

Fig. 3-The variation of $\ln \left[t_{\mathrm{f}}\right]-Q_{\mathrm{c}} / \mathrm{RT}-\delta_{0} \mathrm{D}$ with $r^{*}$ when $(a) k^{*}=0$ and $\delta_{0}=14.507$ with $Q_{\mathrm{c}} \approx 306 \mathrm{kJmol}^{-1}$ and $D=0$ when $\sigma / \sigma_{\mathrm{TS}}>0.41$ and $Q_{\mathrm{c}} \approx 182 \mathrm{kJmol}^{-1}$ with $D=1$ otherwise $(b) k^{*}=1$ and $\delta_{0}=15.384$ with $Q_{\mathrm{c}} \approx 305 \mathrm{kJmol}^{-1}$ and $D=0$ when $\sigma / \sigma_{\mathrm{TS}}>0.38$ and $Q_{\mathrm{c}} \approx 181$ $\mathrm{kJmol}^{-1}$ with $D=1$ otherwise $(c) k^{*}=0.7$ and $\delta_{0}=14.748$ with $Q_{\mathrm{c}} \approx 306 \mathrm{kJmol}^{-1}$ and $D=0$ when $\sigma / \sigma_{\mathrm{TS}}>0.41$ and $Q_{\mathrm{c}} \approx 182 \mathrm{kJmol}^{-1}$ with $D=1$ otherwise. 
5.754E-05 and $\beta_{\mathrm{w}}=1 / 3.737=0.2676$. So these activation energies are not dissimilar to that obtained when using the Wilshire model. The estimated parameters shown in Eq. [11] are based on all the experimental failure times. All the parameters are statistically significant at the 5 pct significance level and the student $t$ values for $\delta_{0}$ through to $\delta_{2}$ also revealed that all these parameters are significantly different from zero at the 5 pct significance level. Consequently, at a normalized stress of 0.38 there is a real change in the value for $\alpha$ and the activation energy, but also and unlike in the Wilshire model, a real change in the slope parameter $\beta$. This structure is shown visually in Figure 3(b), where the fitted line has a steeper slope after the break point. The overall $R^{2}$ value of 97.08 pct is a little higher than that associated with the Wilshire model.

\section{The General Model}

Figure 4 plots the value for $\chi^{2}$ in Eq. [9] for different values of $k^{*}$ and as can be seen the value for $k^{*}$ most supported by the data is 0.7 (and so $k=2.041$ ). This corresponds to neither the Wilshire nor the model by Yang et al. Further, based on the 5 pct significance level it can be concluded that values for $k^{*}$ less than $0.45(k$ more than 4.938) and more than 1.1 ( $k$ less than 1.1) are not supported by the experimental data. Thus, for this material, the Wilshire model is not consistent with the experimental data, while the model by Yang et al. is only just supported by the data, with the general model being most supported by the data when $k=2.041$. Using this value for $k$ and using the estimation technique described above, resulted in the largest $R^{2}$ value being associated with $\sigma_{1}^{\mathrm{c}}=0.41$ - the same as when using the Wilshire model -withIf $\sigma / \sigma_{\mathrm{TS}}>0.41$

$$
\begin{aligned}
& \ln \left[t_{\mathrm{f}}\right]=\underset{\{-10.53\}}{-23.971}+6.413 \ln \left[2.041\left(\frac{\sigma}{\sigma_{\mathrm{TS}}}\right)^{-\frac{1}{2.041}}-2.041\right] \\
& +305,735[1 / R T] .
\end{aligned}
$$

If $\sigma / \sigma_{\mathrm{TS}} \leq 0.41$

$$
+\underset{\{6.92\}}{+181,735[1 / T] .}
$$

The parameter estimates shown in Eq. [12] imply that when $\sigma / \sigma_{\mathrm{TS}}>0.41, \alpha=\exp (-23.971)=3.886 \mathrm{E}-11, \beta=$ $1 / 6.413=0.1559$ with an activation energy of 306 $\mathrm{kJmol}^{-1}$. But at a normalized stress below 0.41 the activation energy drops to $182 \mathrm{kJmol}^{-1}$ with $\alpha=$ $\exp (-9.223)=9.874 \mathrm{E}-05$ and $\beta=1 / 5.164=0.1936$. So these activation energies are not dissimilar to that obtained when using the Wilshire model and the model by Yang et al. The estimated parameters shown in

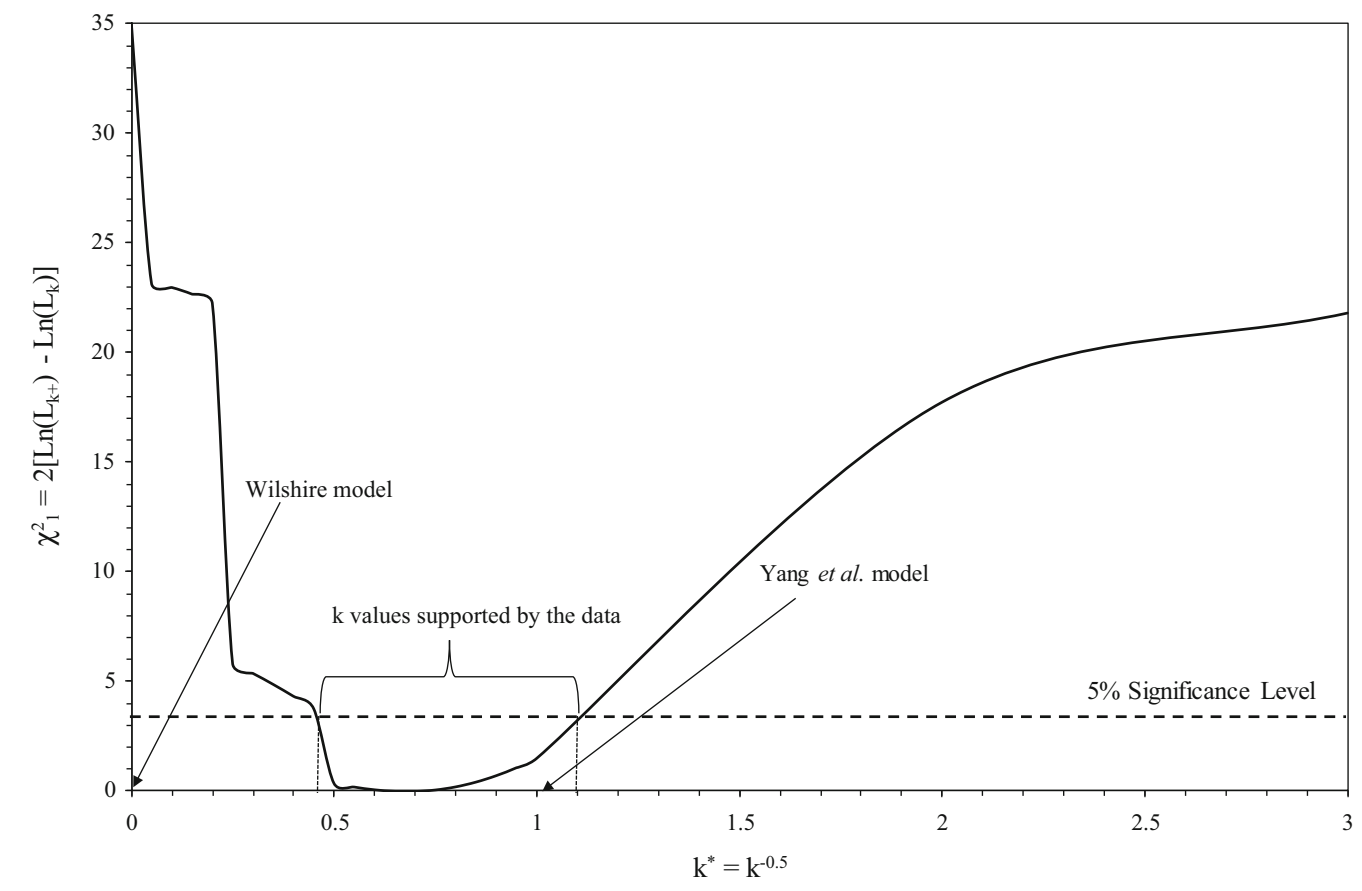

Fig. 4-Variation in the relative log-likelihood function with values for $k^{*}$. 
Eq. [12] are based on all the experimental failure times. All the parameters are statistically significant at the 5 pct significance level and the student $t$ values for $\delta_{0}$ through to $\delta_{2}$ also revealed that all these parameters are significantly different from zero at the 5 pct significance level. Consequently, at a normalized stress of 0.41 there is a real change in the value for $\alpha$ and the activation energy, but also and unlike in the Wilshire model, a real change in the slope parameter $\beta$. This structure is shown visually in Figure 3(c), where the fitted line has a steeper slope after the break point. The overall $R^{2}$ value of 97.16 pct is a little higher than that associated with the Wilshire model.

\section{Deformation Mechanisms Behind the General Model}

Whittaker et al. ${ }^{[19]}$ have found for this material that the processes responsible for creep change at stresses above and below the yield stress. They found that when the stress exceeds the yield stress, creep occurs by movement of new dislocations generated during the plastic component of the initial strain on loading $\left(\varepsilon_{0}\right)$, whereas creep takes place by grain boundary zone deformation when fully elastic $\varepsilon_{0}$ values only are recorded when the stress is below the yield stress. This is confirmed by the results of this paper, where below a normalized stress of 0.41 , all the test stresses are below the 0.2 pet proof stresses (that are approximately equal to the yield stress) shown in Table I. This suggests that for these materials all creep models based on the normalized stress should be of the partitioned variety with one break around the yield stress.

Whittaker et al. ${ }^{[19]}$ further found that under all stress/ temperature conditions, failure occurred by cavitation. If cavity development is strain controlled, then the creep life is determined principally by boundary zone deformation. Assuming that a comparable level of grain boundary zone deformation is necessary to cause failure, the contribution of grain deformation to the overall creep rate decreases with decreasing stress. If this is the case then this should be accompanied by a decrease in the strain at rupture as well. This is what is actually observed in creep data sheet $14 \mathrm{~B}^{[18]}$ where, for example, at a temperature of $1023 \mathrm{~K}$ the percentage elongation at rupture drops from 88 pct as a normalized stress of 0.3 to just 45 pet at a normalized stress of 0.1 . The reader is referred to Whittaker et al. ${ }^{[19]}$ for further details on creep mechanisms and microstructure.

\section{E. Predictive Performance}

Using the general model yields similar estimates of the activation energy to those obtained using the model by Yang et al. or Wilshire-but it is the model most supported by the data. It is then interesting to discover if this fact results in better life time predictions. To assess this, the parameters of all the three modes shown above were re-estimated using data only up to 5000 hours and the resulting equations were then used to predict the failure times for all those specimens that had a life in excess of 5000 hours. This acid or blindfold test of extrapolation was assessed using the root mean percentage squared error (RMPSE) and Theil's ${ }^{[20]}$ inequality coefficient $U$, both defined as

$$
\begin{aligned}
& \text { RMPSE }=\sqrt{\frac{\sum_{i=1}^{n}\left(\ln \left[t_{\mathrm{f}}\right]_{i}-\ln \left[t_{\mathrm{f}}\right]_{i}^{\mathrm{p}}\right)^{2}}{n}} \\
& \cong \sqrt{\frac{\sum_{i=1}^{n}\left(\left[\left[t_{\mathrm{f}}\right]_{i}-\left[t_{\mathrm{f}}\right]_{i}^{\mathrm{p}}\right] /\left[t_{\mathrm{f}}\right]_{i}^{\mathrm{p}}\right)^{2}}{n}} \\
& U=\frac{\sqrt{\frac{\sum_{i=1}^{n}\left(\left[\mathrm{t}_{\mathrm{f}}\right]_{i}-\left[t_{\mathrm{f}}^{\mathrm{p}}\right]_{i}^{2}\right.}{n}}}{\sqrt{\frac{1}{n} \sum_{i=1}^{n}\left(\left[t_{\mathrm{f}}\right]_{i}\right)^{2}}+\sqrt{\frac{1}{n} \sum_{i=1}^{n}\left(\left[t_{\mathrm{f}}\right]_{i}^{\mathrm{p}}\right)^{2}}}
\end{aligned}
$$

where $\ln \left[t_{\mathrm{f}}\right]_{i}^{\mathrm{p}}$ is the value predicted for $\ln \left[t_{\mathrm{f}}\right]_{i}$ and $\left[t_{\mathrm{f}}\right]_{i}^{\mathrm{p}}$ is the value predicted for $\left[t_{\mathrm{f}}\right]_{i}$ by one of the above three creep models. In Eq. [13a], the approximation comes about because the difference between the predicted and actual $\log$ failure time is approximately equal to the percentage difference between the actual and predicted failure times (with the approximation being better the smaller the percentage difference). In Eq. [13b], the numerator is the average of the squared differences between the actual and predicted failure times. The denominator of Eq. [13b] scales $U$ to fall between 0 and 1. If $U=0$, then $\left[t_{\mathrm{f}}\right]_{i}-\left[t_{\mathrm{f}}\right]_{i}^{\mathrm{p}}=0$ for all i (i.e., over all the different test conditions) and the model is a perfect predictor of the creep properties under analysis. If $U=$ 1 , the predictive performance of the creep model is as bad as it could possibly be. Hence $U$ measures predictive accuracy in relative terms.

The results of applying Eq. [13] to the experimental data set are shown in Table III. The greater log likelihood and $R^{2}$ values that are associated with the model by Yang et al. (relative to the Wilshire model) translate to a lower RMPSE over all test temperatures: 46.49 pct for the model by Yang et al. compared to 53.8 pet for the Wilshire model. As the $\mathrm{k}$ value for the general model is not to different to that for the model by Yang et al., the RMPSE for these two models are virtually the same. The same picture emerges when looking at Theil's U statistic. The general model and that by Yang et al. have very similar U statistics, suggesting they have very similar and good predictive performance $(U=0$ implies a perfect model and $U=1$ is the poorest performance possible). The Wilshire model has a much higher $U$ value.

Table III also reveals some interesting differences in predictive performance at specific temperatures. Based on a comparison of Theil's U, all models predict failure times best at a mid-temperature of $973 \mathrm{~K}$, with the general model performing slightly better than the other two nested models. All models predict failure times worst at the temperatures of $873 \mathrm{~K}$ and $1023 \mathrm{~K}$. At 873 $\mathrm{K}$ the general model is the better of the three models, while at $1023 \mathrm{~K}$ the model by Yang et al. is the better performing one, with the general model performing much better than the Wilshire model $(U=0.3<0.36)$ but about as well as the model by Yang et al. Actually, at the highest two temperature, the model by Yang et al. has better $U$ statistic. 
Table III. Predictive Performance of Various Models of Creep Life

\begin{tabular}{|c|c|c|c|c|c|c|}
\hline \multirow{2}{*}{$\begin{array}{l}\text { Model } \\
\text { Temperature (K) }\end{array}$} & \multicolumn{2}{|c|}{$k^{*}=1$ (Yang et al.) } & \multicolumn{2}{|c|}{$k^{*}=0$ (Wilshire $)$} & \multicolumn{2}{|c|}{$k^{*}=0.70($ General $)$} \\
\hline & RMPSE (Pct) & $\mathrm{U}$ & RMPSE (Pct) & $U$ & RMPSE (Pct) & $U$ \\
\hline 873 & 62.85 & 0.31 & 76.58 & 0.36 & 60.05 & 0.30 \\
\hline 923 & 58.90 & 0.29 & 62.27 & 0.31 & 58.39 & 0.29 \\
\hline 973 & 30.72 & 0.15 & 32.81 & 0.15 & 29.39 & 0.13 \\
\hline 1023 & 45.97 & 0.28 & 51.51 & 0.41 & 45.82 & 0.33 \\
\hline 1073 & 45.01 & 0.23 & 56.01 & 0.35 & 46.69 & 0.28 \\
\hline All & 46.49 & 0.25 & 53.80 & 0.39 & 46.53 & 0.27 \\
\hline
\end{tabular}

The RMPSE is given by Eq. [13a] and $U$ is given by Eq. [13b].

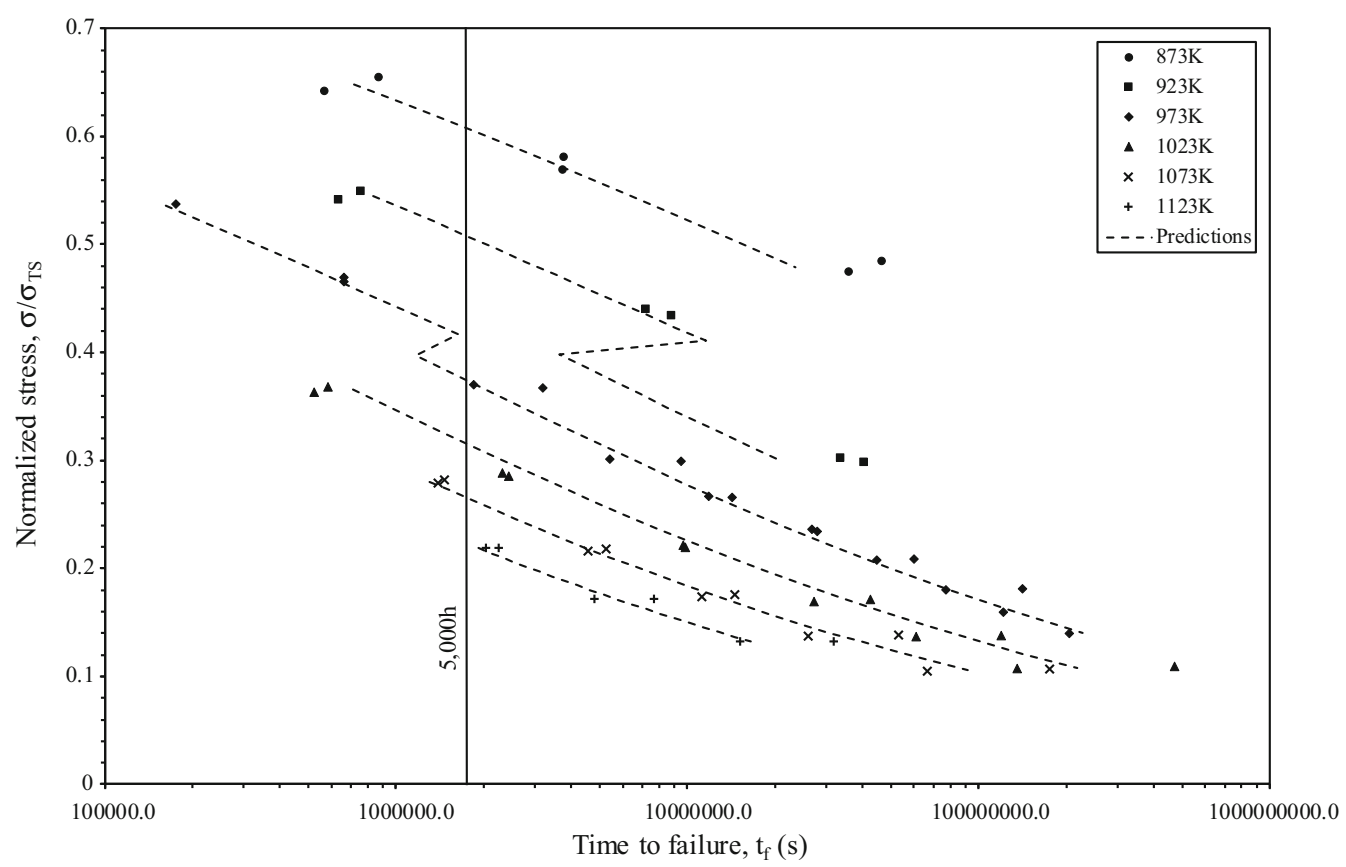

Fig. 5- Stress and temperature dependency of creep life, together with the predicted life times produced by the generalized creep model.

The predictive performance shown in Table III can be seen in Figure 5 where the predicted curves fit the experimental failure times above 5000 hours much better at temperatures above $923 \mathrm{~K}$. The zig-zag nature of the isothermal prediction curves reflects the change in the activation energy at a normalized stress of around 0.41 . It should be noted that this is a model simplification in that in reality the deformation mechanism is likely to gradually change around this critical normalized stress leading to smooth isothermal lines.

\section{CONCLUSIONS}

This paper develops a new parametric creep model that extends the Wilshire and Yang et al. models by having them as restricted or special cases of this new generalized model. These models were then applied to data obtained by NIMS ${ }^{[18]}$ on $316 \mathrm{H}$ stainless steel where blind tests of extrapolative capability for times to failure exceeding 5000 hours were carried out. The main findings from this approach were:
1. There is a large and statistically significant change in the activation energy for $316 \mathrm{H}$ stainless steel at a normalized stress of 0.41 (from around $306 \mathrm{kJmol}^{-1}$ to around $182 \mathrm{kJmol}^{-1}$ ).

2. This change is attributed to stress test conditions being below the yield stress when the normalized stress is below 0.41 , and so there is a change away from dislocation movement within grains to movement within grain boundaries - where the activation energy is considerable lower.

3. The Wilshire model ${ }^{[3]}$ was found to be incompatible with the experimental data at the 5 pct significance level, and while the model by Yang et al. ${ }^{[4]}$ was compatible with the data, it was not a model that was most supported by the data. Such a model was the generalized equation developed in this paper with $k \approx 2$.

4. The Wilshire model ${ }^{[3]}$ produced life time predictions at test conditions leading to lives in excess of 5000 hours that were less accurate than those obtained from the generalized model and the model put forward by Yang et al. ${ }^{[4]}$ Over all test temperatures 
the generalized model produced the most accurate life time predictions.

Areas for future work include the application of this new generalized model to other materials within the NIMS creep data base to see if a $k$ value of around 2 is appropriate for these materials as well. It may turn out to be the case that $k$ (like the Monkman-Grant parameter M) is materials specific. When then comparing the predictive accuracy of this new model with existing models in the literature, it would be important to use the same number of implied creep regimes and use the same data sets/test conditions.

\section{OPEN ACCESS}

This article is distributed under the terms of the Creative Commons Attribution 4.0 International License (http://creativecommons.org/licenses/by/4.0/), which permits unrestricted use, distribution, and reproduction in any medium, provided you give appropriate credit to the original author(s) and the source, provide a link to the Creative Commons license, and indicate if changes were made.

\section{REFERENCES}

1. ASME Boiler and Pressure Vessel Committee on Materials: Boiler and Pressure Vessel Code, section II-materials, ASME, New York, 2004.

2. D. Allen and S. Garwood: Energy Materials-Strategic Research Agenda, 2007. Available online: http://www.matuk.co.uk/docs/1 StrategicResearchAgenda\%20FINAL.pdf. Accessed 28 May 2019.
3. B. Wilshire and A.J. Battenbough: Mater. Sci. Eng. A, 2007, vol. 443, pp. 156-66.

4. M. Yang, Q. Wang, X.L. Song, J. Jia, and Z.D. Xiang: Metall. Mater. Trans. A, 2016, vol. 48A (7), pp. 3479-87.

5. H. Eyring: Basic Chemical Kinetics, Wiley, New York, 1980.

6. F.C. Monkman and N.J. Grant: Proc. Am. Soc. Test. Mater., 1956, vol. 56, pp. 593.

7. I.I. Trunin, N.G. Golobova and E.A. Loginov: In: Proc. 4th Int. Symp. On Heat-Resistant Metallic Materials, Mala Fatravol, 1971, vol. 168 , CSSR.

8. F.R. Larson and J.A. Miller: Trans. ASME, 1952, vol. 74, pp. 765-75.

9. L.R. Orr, O.D. Sherby, and J.E. Dorn: Trans. ASME, 1954, vol. 46, p. 113.

10. S. S. Manson and U. Muraldihan: EPRI CS-3171, Research project 638-1, 1983.

11. S. S. Manson and A. M. Haferd: NACA TN 2890, 1953.

12. S.S. Manson and W.F. Brown: Proc. ASTM, 1953, vol. 53, pp. $683-719$.

13. B. Wilshire and P.J. Scharning: Mater. Sci. Technol., 2008, vol. 24 (1), pp. 1-9.

14. B. Wilshire and M. Whittaker: Mater. Sci. Technol., 2011, vol. 27 (3), pp. 642-47.

15. M. Evans: Appl. Math. Model., 2016, vol. 40 (23-24), pp. $10342-59$.

16. M.T. Whittaker, W. Harrison, C. Den, C. Rae, and S. Williams: Materials, 2017, vol. 10 (1), p. 61.

17. Creep datasheets in MatNavi database (National Institute of Materials Science of Japan). www.nims.go.jp/matnavi. Accessed May 2019.

18. NIMS Creep Data Sheet No.14B: Data Sheets on the Elevated-Temperature Properties of $18 \mathrm{Cr}-12 \mathrm{Ni}-\mathrm{Mo}$ Stainless Steel Plates for Reactor Vessels (SUS 316HP), 2nd ed, National Research Institute for Metals, Tokyo, Japan, 1988.

19. M.T. Whittaker, M. Evans, and B. Wilshire: Mater. Sci. Eng. A, 2012, vol. 552, pp. 145-50.

20. H. Theil: Applied Economic Forecasts, North Holland, Amsterdam, 1966.

Publisher's Note Springer Nature remains neutral with regard to jurisdictional claims in published maps and institutional affiliations. 